Tôhoku Math. Journ.

29 (1977), 439-448.

\title{
ON THE ABSOLUTE NÖRLUND SUMMABILITY \\ FACTORS OF FOURIER SERIES
}

\author{
Kōsi Kanno and Yasuo OKuYama
}

(Received May 10, 1976)

1. Let $\left\{s_{n}\right\}$ denote the $n$-th partial sum of a given infinite series $\sum a_{n}$. Let $\left\{p_{n}\right\}$ be a sequence of constants, real or complex, and let

$$
P_{n}=p_{0}+p_{1}+\cdots+p_{n} ; P_{-k}=p_{-k}=0, \text { for } k \geqq 1 \text {. }
$$

The sequence $\left\{t_{n}\right\}$, given by

$$
t_{n}=\frac{1}{P_{n}} \sum_{k=0}^{n} p_{n-k} s_{k}=\frac{1}{P_{n}} \sum_{k=0}^{n} P_{n-k} a_{k}, \quad\left(P_{n} \Longleftarrow 0\right),
$$

defines the Nörlund means of the sequence $\left\{s_{n}\right\}$ generated by the sequence $\left\{p_{n}\right\}$.

Then, the series $\sum a_{n}$ is said to be summable $\left|N, p_{n}\right|$, if the sequence $\left\{t_{n}\right\}$ is of bounded variation, that is, the series

$$
\sum_{n=1}^{\infty}\left|t_{n}-t_{n-1}\right|
$$

is convergent.

In the special cases in which $p_{n}=\Gamma(n+\alpha) / \Gamma(\alpha) \Gamma(n+1), \alpha>0$, and $p_{n}=1 /(n+1)$, summability $\left|N, p_{n}\right|$ are the same as the summability $|C, \alpha|$ and the absolute harmonic summability, respectively.

Let $f(t)$ be a periodic function with period $2 \pi$ and integrable $(L)$ over $(-\pi, \pi)$. We assume without any loss of generality that the Fourier series of $f(t)$ is given by

$$
\sum_{n=1}^{\infty}\left(a_{n} \cos n t+b_{n} \sin n t\right)=\sum_{n=1}^{\infty} A_{n}(t)
$$

and $\int_{-\pi}^{\pi} f(t) d t=0$.

We use the notations

$$
\begin{aligned}
& \varphi_{x}(t)=\varphi(t)=\frac{1}{2}\{f(x+t)+f(x-t)\} ; \\
& \triangle \lambda(n)=\lambda(n)-\lambda(n+1) ; \\
& \frac{d \lambda(t)}{d t}=\lambda^{\prime}(t) ;
\end{aligned}
$$

[x] denotes the integral part of real number $x$. 
2. Recently, one of the present authors [10] proved the following theorem, which is a generalization of theorems due to M. Izumi and S. Izumi [3] and K. Kanno [5].

THEOREM A. Let $\left\{p_{n}\right\}$ be non-negative and non-increasing. Suppose that $\{\mu(n)\}$ is a positive bounded sequence and $\lambda(t), t>0$, is a positive non-decreasing function such that $\{\lambda(n) \mu(n) /(n+1)\}$ is non-increasing.

If the conditions

$$
\sum_{k=n}^{\infty} \frac{\lambda(k) \mu(k)}{k P_{k}}=O\left(\frac{\lambda(n)}{P_{n}}\right), \quad n=1,2, \cdots
$$

and

$$
\int_{0}^{\pi} \lambda(C / t)|d \varphi(t)|<\infty
$$

hold for some constant $C(>2 \pi)$, then the series

$$
\sum_{n=1}^{\infty} \lambda(n) \mu(n) A_{n+1}(t)
$$

is summable $\left|N, p_{n}\right|$, at $t=x$.

If we replace the condition (2.2) by the condition

$$
\lambda(C / t) \varphi(t) \in B V(0, \pi) \text { for a constant } C>\pi,
$$

then next theorems are known.

THEOREM B (M. and S. Izumi [4]). Let $\left\{p_{n}\right\}$ be a positive non-increasing sequence and $\alpha \geqq 0$.

If the conditions

$$
\sum_{k=n}^{\infty} \frac{1}{k P_{k}}=O\left(\frac{(\log n)^{\alpha}}{P_{n}}\right) \text { for all } n \geqq 1
$$

and

$$
\varphi(t)(\log C / t)^{\alpha} \in B V(0, \pi) \text { for a constant } C(>\pi)
$$

hold, then the series $\sum_{n=1}^{\infty} A_{n}(t)$ is summable $\left|N, p_{n}\right|$, at $t=x$.

This theorem is a generalization of the theorems of T. Singh [13] and O. P. Varshney [15].

Theorem C (M. Mudiraj [9]). Let $\left\{p_{n}\right\}$ be a positive non-increasing sequence such that

$$
\left\{\triangle\left(P_{n} / p_{n}\right)\right\} \text { is bounded }
$$

and 


$$
P_{m} / P_{n}=O\left\{(m / n)^{\alpha}\right\}, \text { where } 0<\alpha \leqq 1, \text { for } m \leqq n,
$$

uniformly in $m, n$.

Let $\{\mu(n)\}$ be a positive non-increasing sequence such that the series $\sum_{n=1}^{\infty} \mu(n) / n$ converges. If

$$
t^{-\alpha} \varphi(t) \in B V(0, \pi),
$$

then the series

$$
\sum_{n=1}^{\infty} n^{\alpha} \mu(n) A_{n}(t)
$$

is summable $\left|N, p_{n}\right|$, at $t=x$.

Theorem $\mathrm{C}$ is an extension of the theorem due to S. M. Mazhar [7] for the case $\beta=0$. Putting $\lambda(t)=t^{\alpha}$, the condition (2.1) is deduced from the condition (2.7) under the hypothesis of $\mu(n)$ in Theorem $\mathrm{C}$.

Now, S. Izumi [2] proved the following theorem.

THEOREM D. Two conditions

$$
(\log 2 \pi / t) f(t) \in B V(0, \pi)
$$

and

$$
\int_{0}^{\pi}(\log 2 \pi / t)|d f(t)|<\infty
$$

are mutually exclusive.

Also, Y. Okuyama [11] proved the following theorem.

THEOREM E. Let $\lambda(t)$ be a non-decreasing function. If the condition

$$
\int_{0}^{\pi} \frac{\lambda^{\prime}(C / t)|\varphi(t)|}{t^{2}} d t<\infty
$$

holds, then the condition (2.2) is equivalent to the condition (2.3).

Thus, by these Theorems $\mathrm{D}$ and $\mathrm{E}$, we see that Theorem $\mathrm{A}$ does not necessarily contain Theorems $\mathrm{B}$ and $\mathrm{C}$.

In this paper, we shall generalize these Theorems $\mathrm{B}$ and $\mathrm{C}$ in the following form.

THEOREM. Let $\left\{p_{n}\right\}$ be non-negative and non-increasing. Suppose that $\lambda(t), t>0$, is a positive non-decreasing function such that $t \lambda^{\prime}(t) / \lambda^{2}(t)$ is non-increasing, $t^{2} \lambda^{\prime}(t) / \lambda^{2}(t)$ is non-decreasing and $\{\lambda(n) \mu(n) / n\}$ is nonincreasing, where $\mu(t), t>0$, is a positive bounded function.

If the conditions 


$$
\begin{gathered}
\sum_{k=1}^{\infty} \frac{\lambda^{\prime}(k) \mu(k)}{\lambda(k)}<\infty, \\
\sum_{k=n}^{\infty} \frac{\lambda(k) \mu(k)}{k P_{k}}=O\left(\frac{\lambda(n)}{P_{n}}\right), n=1,2, \cdots
\end{gathered}
$$

and

$$
\lambda(C / t) \varphi(t) \in B V(0, \pi) \text { for a constant } C(>\pi)
$$

hold, then the series

$$
\sum_{n=1}^{\infty} \lambda(n) \mu(n) A_{n+1}(t)
$$

is summable $\left|N, p_{n}\right|$, at $t=x$.

If we put in our theorem $\lambda(t)=(\log t)^{\alpha}$ and $\mu(k)=1 /(\log k)^{\alpha}(\alpha \geqq 0)$, then $t \lambda^{\prime}(t) / \lambda^{2}(t)=\alpha /(\log t)^{\alpha+1}$ is non-increasing and $t^{2} \lambda^{\prime}(t) / \lambda^{2}(t)=\alpha t /(\log t)^{\alpha+1}$ is non-decreasing. Further, we can easily see that

$$
\sum_{k=2}^{\infty} \frac{\lambda^{\prime}(k) \mu(k)}{\lambda(k)}=\sum_{k=2}^{\infty} \frac{\alpha}{k(\log k)^{1+\alpha}}<\infty
$$

and

$$
\sum_{k=n}^{\infty} \frac{\lambda(k) \mu(k)}{k P_{k}}=\sum_{k=n}^{\infty} \frac{1}{k P_{k}}=O\left(\frac{\lambda(n)}{P_{n}}\right)=O\left(\frac{(\log n)^{\alpha}}{P_{n}}\right) .
$$

Thus our theorem contains Theorem B.

Similarly, if we put $\lambda(t)=t^{\alpha}$ and $p_{n}=\Gamma(n+\alpha) / \Gamma(\alpha) \Gamma(n+1)(0 \leqq \alpha \leqq 1)$ then Theorem $\mathrm{C}$ is deduced from our theorem.

3. We need some lemmas for the proof of our theorem.

Lemma 1 (H. P. Dikshit [1]). Let $\left\{p_{n}\right\}$ be a given sequence, then for any $x$, we have

$$
(1-x) \sum_{k=m}^{n} p_{k} x^{k}=p_{m} x^{m}-p_{n} x^{n+1}-\sum_{k=m}^{n-1} \triangle p_{k} x^{k+1}
$$

where $n \geqq m \geqq 0$.

LEMMA 2 (L. McFadden [8]). If $\left\{p_{n}\right\}$ is non-negative and non-increasing, then for $0 \leqq a \leqq b<\infty, 0 \leqq t \leqq \pi$, and for any $n$, we have

$$
\left|\sum_{k=a}^{b} p_{k} \exp (i(n-k) t)\right| \leqq A P_{[1 / t]},
$$

where $A$ is a positive constant, not necessarily the same at each occurences.

Lемма 3. Let $\lambda(t), t>0$, be a positive non-decreasing function. If 
$t \lambda^{\prime}(t) / \lambda^{2}(t)$ is non-increasing and $t^{2} \lambda^{\prime}(t) / \lambda^{2}(t)$ is non-decreasing, then we have

$$
\left|\int_{0}^{\pi} \frac{\cos k t}{\lambda(C / t)} d t\right| \leqq A \frac{\lambda^{\prime}(k)}{\lambda^{2}(k)} \text { for a constant } C(>\pi) .
$$

Proof. By an integration by parts, we have

$$
\begin{gathered}
J=\int_{0}^{\pi} \frac{\cos k t}{\lambda(C / t)} d t=\left[\frac{\sin k t}{k \lambda(C / t)}\right]_{0}^{\pi}-\frac{1}{k} \int_{0}^{\pi} \frac{d}{d t}\left\{\frac{1}{\lambda(C / t)}\right\} \sin k t d t \\
=\frac{C}{k} \int_{0}^{\pi} \frac{\lambda^{\prime}(C / t)}{t^{2} \lambda^{2}(C / t)} \sin k t d t .
\end{gathered}
$$

Since $\lambda^{\prime}(C / t) / t \lambda^{2}(C / t)$ is non-decreasing and $\lambda^{\prime}(C / t) / t^{2} \lambda^{2}(C / t)$ is non-increasing, we obtain

$$
\begin{aligned}
|J| & \leqq \frac{C}{k} \int_{0}^{\pi / k} \frac{\lambda^{\prime}(C / t)}{t^{2} \lambda^{2}(C / t)} \sin k t d t \\
& =\frac{C}{k} \int_{0}^{\pi / k} \frac{\lambda^{\prime}(C / t)}{t \lambda^{2}(C / t)} \frac{\sin k t}{t} d t \\
& \leqq \frac{C}{k} \frac{k \lambda^{\prime}\left(\frac{C}{\pi} k\right)}{\pi \lambda^{2}\left(\frac{C}{\pi} k\right)} \int_{0}^{\pi / k} \frac{\sin k t}{t} d t \\
& \leqq A \frac{\lambda^{\prime}(k)}{\lambda^{2}(k)}
\end{aligned}
$$

Hence we complete the proof of Lemma 3.

4. Proof of Theorem. By (1.1), we have

$$
t_{n}=\frac{1}{P_{n}} \sum_{k=0}^{n} P_{n-k} \lambda(k) \mu(k) A_{k+1}(x)
$$

where

$$
A_{k+1}(x)=\frac{2}{\pi} \int_{0}^{\pi} \varphi(t) \cos (k+1) t d t .
$$

Hence we have by (4.1) and (4.2)

$$
\begin{aligned}
t_{n}-t_{n-1} & =\sum_{k=1}^{n}\left(\frac{P_{n-k}}{P_{n}}-\frac{P_{n-k-1}}{P_{n-1}}\right) \lambda(k) \mu(k) A_{k+1}(x) \\
& =\frac{2}{\pi} \int_{0}^{\pi} \varphi(t)\left\{\sum_{k=1}^{n}\left(\frac{P_{n-k}}{P_{n}}-\frac{P_{n-k-1}}{P_{n-1}}\right) \lambda(k) \mu(k) \cos (k+1) t\right\} d t
\end{aligned}
$$

Now, we put 


$$
g(t)=\lambda(C / t) \varphi(t) \quad \text { for } \quad 0<t \leqq \pi .
$$

Then, by (4.2) and an integration by parts, we have

$$
A_{k+1}(x)=\frac{2}{\pi} g(\pi) \int_{0}^{\pi} \frac{\cos (k+1) t}{\lambda(C / t)} d t-\frac{2}{\pi} \int_{0}^{\pi} d g(t) \int_{0}^{t} \frac{\cos (k+1) u}{\lambda(C / u)} d u .
$$

Putting $\tau=[C / 2 t]$, we have by (4.3) and (4.4)

$$
\begin{aligned}
\sum_{n=1}^{\infty} \mid t_{n} & -t_{n-1}|<A| g(\pi)\left|\sum_{n=1}^{\infty}\right| \sum_{k=1}^{n}\left(\frac{P_{n-k}}{P_{n}}-\frac{P_{n-k-1}}{P_{n-1}}\right) \lambda(k) \mu(k) \int_{0}^{\pi} \frac{\cos (k+1) t}{\lambda(C / t)} d t \mid \\
& +A \int_{0}^{\pi}|d g(t)|\left\{\sum_{n=1}^{2 \tau+1}\left|\sum_{k=1}^{n}\left(\frac{P_{n-k}}{P_{n}}-\frac{P_{n-k-1}}{P_{n-1}}\right) \lambda(k) \mu(k) \int_{0}^{t} \frac{\cos (k+1) u}{\lambda(C / u)} d u\right|\right\} \\
& +A \int_{0}^{\pi}|d g(t)|\left\{\sum_{n=2 \tau+2}^{\infty}\left|\sum_{k=1}^{n}\left(\frac{P_{n-k}}{P_{n}}-\frac{P_{n-k-1}}{P_{n-1}}\right) \lambda(k) \mu(k) \int_{0}^{t} \frac{\cos (k+1) u}{\lambda(C / u)} d u\right|\right\} \\
& =I_{1}+I_{2}+I_{3},
\end{aligned}
$$

say. By Lemma 3, we obtain

$$
\begin{aligned}
I_{1} & \leqq A \sum_{n=1}^{\infty} \sum_{k=1}^{n}\left(\frac{P_{n-k}}{P_{n}}-\frac{P_{n-k-1}}{P_{n-1}}\right) \lambda(k) \mu(k) \frac{\lambda^{\prime}(k)}{\lambda^{2}(k)} \\
& =A \sum_{k=1}^{\infty} \frac{\lambda^{\prime}(k) \mu(k)}{\lambda(k)} \sum_{n=k}^{\infty}\left(\frac{P_{n-k}}{P_{n}}-\frac{P_{n-k-1}}{P_{n-1}}\right) \\
& \leqq A \sum_{k=1}^{\infty} \frac{\lambda^{\prime}(k) \mu(k)}{\lambda(k)}<\infty,
\end{aligned}
$$

because the sequence $\left\{P_{n-k} / P_{n}\right\}(k \geqq 1)$ is bounded, non-decreasing and the hypothesis (2.11) holds.

Since

$$
\left|\int_{0}^{t} \frac{\cos (k+1) u}{\lambda(C / u)} d u\right| \leqq \frac{t}{\lambda(C / t)}
$$

we have

$$
\begin{aligned}
I_{2} & \leqq A \int_{0}^{\pi}|d g(t)|\left\{\frac{t}{\lambda(C / t)} \sum_{n=1}^{2 \tau+1} \sum_{k=1}^{n}\left(\frac{P_{n-k}}{P_{n}}-\frac{P_{n-k-1}}{P_{n-1}}\right) \lambda(k) \mu(k)\right\} \\
& =A \int_{0}^{\pi}|d g(t)|\left\{\frac{t}{\lambda(C / t)} \sum_{k=1}^{2 \tau+1} \lambda(k) \mu(k) \sum_{n=k}^{2 \tau+1}\left(\frac{P_{n-k}}{P_{n}}-\frac{P_{n-k-1}}{P_{n-1}}\right)\right\} \\
& \leqq A \int_{0}^{\pi}|d g(t)|\left\{\frac{t}{\lambda(C / t)} \sum_{k=1}^{2 \tau+1} \lambda(k) \mu(k)\right\} \\
& \leqq A \int_{0}^{\pi}|d g(t)|\left\{\frac{t}{\lambda(C / t)} \cdot \lambda(2 \tau) \cdot 2 \tau\right\} \\
& \leqq A \int_{0}^{\pi}|d g(t)|<\infty
\end{aligned}
$$


by virtue of the hypothesis that $\lambda(k)$ is non-decreasing and $\mu(k)$ is bounded.

By an integration by parts, we have

$$
\int_{0}^{t} \frac{\cos (k+1) u}{\lambda(C / u)} d u=\frac{\sin (k+1) t}{(k+1) \lambda(C / t)}+\frac{C}{(k+1)} \int_{0}^{t} \frac{\lambda^{\prime}(C / t)}{t^{2} \lambda^{2}(C / t)} \sin (k+1) t d t .
$$

Thus we obtain

$$
\begin{aligned}
I_{3} & \leqq A \int_{0}^{\pi}|d g(t)|\left\{\frac{1}{\lambda(C / t)} \sum_{n=2 \tau+2}^{\infty}\left|\sum_{k=1}^{n}\left(\frac{P_{n-k}}{P_{n}}-\frac{P_{n-k-1}}{P_{n-1}}\right) \lambda(k) \mu(k) \frac{\sin (k+1) t}{(k+1)}\right|\right\} \\
& +A \int_{0}^{\pi}|d g(t)|\left\{\sum_{n=2 \tau+2}^{\infty}\left|\sum_{k=1}^{n}\left(\frac{P_{n-k}}{P_{n}}-\frac{P_{n-k-1}}{P_{n-1}}\right) \frac{\lambda(k) \mu(k)}{(k+1)}\right| \int_{0}^{t} \frac{\lambda^{\prime}(C / t)}{t^{2} \lambda^{2}(C / t)} \sin (k+1) t d t \mid\right\} \\
& =I_{31}+I_{32},
\end{aligned}
$$

say. By the same method as that used by Y. Okuyama [10], we have

$$
\sum_{n=2 \tau+2}^{\infty}\left|\sum_{k=1}^{n}\left(\frac{P_{n-k}}{P_{n}}-\frac{P_{n-k-1}}{P_{n-1}}\right) \lambda(k) \mu(k) \frac{\sin (k+1) t}{(k+1)}\right|=O(\lambda(C / t)) .
$$

Thus we proved the finiteness of $I_{31}$. On the other hand, by the similar estimation as $|J|$ in the proof of Lemma 3, we have

$$
\left|\int_{0}^{t} \frac{\lambda^{\prime}(C / t)}{t^{2} \lambda^{2}(C / t)} \sin (k+1) t d t\right| \leqq A \frac{k \lambda^{\prime}(k)}{\lambda^{2}(k)} \quad \text { for } \quad 0<t \leqq \pi .
$$

Thus we have

$$
\begin{aligned}
I_{32} & \leqq A \int_{0}^{\pi}|d g(t)|\left\{\sum_{n=2 \tau+2}^{\infty} \sum_{k=1}^{n}\left(\frac{P_{n-k}}{P_{n}}-\frac{P_{n-k-1}}{P_{n-1}}\right) \frac{\lambda(k) \mu(k)}{(k+1)} \frac{k \lambda^{\prime}(k)}{\lambda^{2}(k)}\right\} \\
& =A \int_{0}^{\pi}|d g(t)|\left\{\sum_{k=1}^{2 \tau+2} \frac{\lambda^{\prime}(k) \mu(k)}{\lambda(k)} \sum_{n=2 \tau+2}^{\infty}\left(\frac{P_{n-k}}{P_{n}}-\frac{P_{n-k-1}}{P_{n-1}}\right)\right. \\
& \left.+\sum_{k=2 \tau+3}^{\infty} \frac{\lambda^{\prime}(k) \mu(k)}{\lambda(k)} \sum_{n=k}^{\infty}\left(\frac{P_{n-k}}{P_{n}}-\frac{P_{n-k-1}}{P_{n-1}}\right)\right\} \\
& \leqq A \int_{0}^{\pi}|d g(t)|\left\{\sum_{k=1}^{\infty} \frac{\lambda^{\prime}(k) \mu(k)}{\lambda(k)}\right\} \\
& \leqq A \int_{0}^{\pi}|d g(t)|<\infty
\end{aligned}
$$

by virtue of the hypothesis (2.11). Thus, by $I_{31}$ and $I_{32}$, we see that $I_{3}$ is finite.

Collecting the above estimations, we have

$$
\sum_{n=1}^{\infty}\left|t_{n}-t_{n-1}\right|<\infty
$$

Therefore, we complete the proof of our theorem. 
5. In this section, we consider some applications of our theorem.

Corollary 1. If $1>\alpha \geqq 0, \beta \geqq 0, \alpha+\beta<1$, and

$$
(\log C / t)^{\beta} \varphi(t) \in B V(0, \pi) \text {, }
$$

then the series

$$
\sum_{n=0}^{\infty} \frac{A_{n}(t)}{\{\log (n+2)\}^{1-\beta}} \quad \text { is summable }\left|N, 1 /(n+2)\{\log (n+2)\}^{\alpha}\right|
$$

at $t=x$.

For $\alpha=\beta=0$, this corollary is due to O.P. Varshney [14].

Corollary 2. If $\alpha>0$ and

$$
(\log C / t) \varphi(t) \in B V(0, \pi),
$$

then the series

$$
\sum_{n=0}^{\infty} A_{n}(t) \quad \text { is summable }\left|N,\{\log (n+2)\}^{\alpha} /(n+2)\right|
$$

at $t=x$.

Corollary 2 is due to M. Izumi and S. Izumi [4]. This corollary does not hold for $\alpha=0$ by Pati's theorem [12], and the case $\alpha=1$ in the corollary is due to O.P. Varshney [15].

CoROllary 3. If

$$
(\log \log C / t)^{\beta} \varphi(t) \in B V(0, \pi) \quad \text { for } \quad 0 \leqq \beta<1,
$$

then the series

$$
\sum_{n=0}^{\infty} \frac{A_{n}(t)}{\log (n+2)\{\log \log (n+2)\}^{1-\beta}} \text { is summable }|N, 1 /(n+2) \log (n+2)|
$$

at $t=x$.

CoRollary 4. If $\alpha>0$ and

$$
(\log \log C / t) \varphi(t) \in B V(0, \pi),
$$

then the series

$$
\sum_{n=0}^{\infty} \frac{A_{n}(t)}{\log (n+2)} \quad \text { is summable }\left|N,\{\log \log (n+2)\}^{\alpha} /(n+2) \log (n+2)\right|
$$

at $t=x$.

Corollary 5. If $\alpha \geqq 0$ and

$$
t^{-\alpha} \varphi(t) \in B V(0, \pi),
$$

then the series 


$$
\sum_{n=1}^{\infty} \frac{n^{\alpha}}{\{\log (n+1)\}^{1+\varepsilon}} A_{n}(t) \text { is summable }|C, \alpha|
$$

at $t=x$, where $\varepsilon>0$.

For Corollary 5, the reader is also referred to K. Matsumoto [6] for the case $\beta=0$.

As these corollaries are similarly proved, we shall prove here only Corollary 1.

Proof of Corollary 1. In our theorem, we put $p_{k}=1 /(k+2)\{\log (k+2)\}^{\alpha}$, $\lambda(t)=\{\log (t+2)\}^{\beta}$ and $\mu(k)=1 / \log (k+2)$. Then we have

$$
\begin{aligned}
& P_{k}=\sum_{l=0}^{k} \frac{1}{(l+2)\{\log (l+2)\}^{\alpha}} \cong\{\log (k+2)\}^{1-\alpha}, \\
& \sum_{k=1}^{\infty} \frac{\lambda^{\prime}(k) \mu(k)}{\lambda(k)} \leqq A \sum_{k=1}^{\infty} \frac{1}{(k+2)\{\log (k+2)\}^{2}}<\infty
\end{aligned}
$$

and

$$
\sum_{k=n}^{\infty} \frac{\lambda(k) \mu(k)}{k P_{k}}=O\left(\frac{1}{\{\log (n+2)\}^{1-\alpha-\beta}}\right)=O\left(\frac{\lambda(n)}{P_{n}}\right) .
$$

Hence we see that all assumptions of our theorem hold. Therefore, the proof is complete.

\section{REFERENCES}

[1] H. P. Dikshit, Absolute summability of a Fourier series with factors, preprint.

[2] S. Izumi, Absolute convergence of some trigonometric series II, Jour. Math. Anal. and Appl., 1(1960), 184-194.

[3] M. Izumi and S. Izumi, Absolute Nörlund summability factor of Fourier series, Proc. Japan Acad., 46 (1970), 642-648.

[4] M. Izumi and S. Izumi, Absolute Nörlund summability of Fourier series of functions of bounded variation, Bull. Austral. Math. Soc., 3 (1970), 111-123.

[5] K. KanNo, On the absolute Nörlund summability of the factored Fourier series, Tôhoku Math. J., 21 (1969), 434-447.

[6] K. Матsumoto, On absolute Cesàro summability of a series related to a Fourier series, Tôhoku Math. J., 8 (1956), 205-222.

[7] S. M. MAzHAR, A theorem on the absolute Cesàro summability of factored Fourier series, Annali Mat. Pura Appl., 59 (1962), 11-26.

[8] L. McFadDEN, Absolute Nörlund summability, Duke Math. J., 9 (1942), 168-207.

[9] M. MudiraJ, Absolute Nörlund summability factors of Fourier series, Rend. Mat., 5 (1972), 603-612.

[10] Y. OKuYama, On the absolute Nörlund summablilty factors of Fourier series, Bull. Austral. Math. Soc., 12 (1975), 9-21.

[11] Y. OKuYama, On the absolute Nörlund summability factors of the conjugate series of a Fourier series, Tôhoku Math. J., 28 (1976), 563-581.

[12] T. PATI, The non-absolute summability of Fourier series by a Nörlund method, Indian 
J. Math., 25 (1961), 197-214.

[13] T. SingH, Absolute Nörlund summablity of Fourier series, Indian J. Math. 6 (1964), 129-136.

[14] O. P. VARShney, On the absolute harmonic summability of a series related to a Fourier series, Proc. Amer. Math. Soc., 10 (1959), 784-789.

[15] O. P. VARShney, On the absolute summability of Fourier series by a Nörlund method, Univ. Roorkee Res. J., 6 (1963), 103-113.

Department of Mathematics

YAMAGATA UNIVERSITY

YAMAGATA, JAPAN

AND

Department of Mathematics

FACULTy OF ENGINEERING

SHINSHU UNIVERSITY

NAGANO, JAPAN 